\title{
Isolation and Characterization of a Novel Pathogenic Strain of Ehrlichia minasensis
}

\author{
Daniel Moura de Aguiar ${ }^{1, *(\mathbb{C})}$, João Pessoa Araújo Junior $\left.{ }^{2} \mathbb{(}\right)$, Luciano Nakazato ${ }^{1}\left(\mathbb{D}\right.$, Emilie Bard $^{3}$, \\ Lisandra Aguilar-Bultet ${ }^{4}$, Fabien Vorimore ${ }^{5}$, Vsevolod Leonidovich Popov ${ }^{6}$, \\ Edson Moleta Colodel ${ }^{1}$ and Alejandro Cabezas-Cruz ${ }^{7, *}$ (i) \\ 1 Veterinary Hospital, Faculty of Veterinary Medicine, Federal University of Mato Grosso State (UFMT), \\ 78060-900 Cuiabá, Brazil; lucnaka@gmail.com (L.N.); moleta@ufmt.br (E.M.C.) \\ 2 Biotechnology Institute (IBTEC), São Paulo State University (UNESP), 18607-440 Botucatu, Brazil; \\ joao.pessoa@unesp.br \\ 3 EPIA, INRA, VetAgro Sup, 63122 Saint Genès Champanelle, France; emilie.bard@inra.fr \\ 4 Department of Infectious Diseases and Hospital Epidemiology, University Hospital Basel, 4031 Basel, \\ Switzerland; lisandritta@gmail.com \\ 5 University Paris-Est, Anses, Animal Health Laboratory, Bacterial Zoonoses Unit, 94706 Maisons-Alfort, \\ France; fabien.vorimore@anses.fr \\ 6 Department of Pathology, University of Texas Medical Branch at Galveston, Galveston, TX 77555, USA; \\ vpopov@utmb.edu \\ 7 UMR BIPAR, INRA, ANSES, Ecole Nationale Vétérinaire d'Alfort, Université Paris-Est, \\ 94700 Maisons-Alfort, France \\ * Correspondence: danmoura@ufmt.br (D.M.d.A.); cabezasalejandrocruz@gmail.com (A.C.-C.)
}

Received: 7 October 2019; Accepted: 1 November 2019; Published: 5 November 2019

\begin{abstract}
The genus Ehrlichia is composed of tick-borne obligate intracellular gram-negative alphaproteobacteria of the family Anaplasmataceae. Ehrlichia includes important pathogens affecting canids (E. canis, E. chaffeensis, and E. ewingii), rodents (E. muris), and ruminants (E. ruminantium). Ehrlichia minasensis, an Ehrlichia closely related to E. canis, was initially reported in Canada and Brazil. This bacterium has now been reported in Pakistan, Malaysia, China, Ethiopia, South Africa, and the Mediterranean island of Corsica, suggesting that E. minasensis has a wide geographical distribution. Previously, E. minasensis was found to cause clinical ehrlichiosis in an experimentally infected calf. The type strain E. minasensis UFMG-EV was successfully isolated from Rhipicephalus microplus ticks and propagated in the tick embryonic cell line of Ixodes scapularis (IDE8). However, the isolation and propagation of E. minasensis strains from cattle has remained elusive. In this study, the E. minasensis strain Cuiabá was isolated from an eight-month-old male calf of Holstein breed that was naturally infected with the bacterium. The calf presented clinical signs and hematological parameters of bovine ehrlichiosis. The in vitro culture of the agent was established in the canine cell line DH82. Ehrlichial morulae were observed using light and electron microscopy within DH82 cells. Total DNA was extracted, and the full genome of the E. minasensis strain Cuiabá was sequenced. A core-genome-based phylogenetic tree of Ehrlichia spp. and Anaplasma spp. confirmed that E. minasensis is a sister taxa of E. canis. A comparison of functional categories among Ehrlichia showed that E. minasensis has significantly less genes in the 'clustering-based subsystems' category, which includes functionally coupled genes for which the functional attributes are not well understood. Results strongly suggest that E. minasensis is a novel pathogen infecting cattle. The epidemiology of this Ehrlichia deserves further attention because these bacteria could be an overlooked cause of tick-borne bovine ehrlichiosis, with a wide distribution.
\end{abstract}

Keywords: anaplasmataceae; Ehrlichia minasensis; bovine ehrlichiosis; DH82; transmission electron microscopy; genome 


\section{Introduction}

The genus Ehrlichia belongs to the family Anaplasmataceae and consists of six recognized species of bacteria: E. canis, E. chaffeensis, E. muris, E. ewingii, E. ruminantium, and E. minasensis [1,2]. Prior to 2010, ehrlichiosis in bovines was mostly associated with infection by E. ruminantium, a bacterium present in the African continent and in some regions of the Caribbean [3,4]. However, in the early 2010s, an ehrlichial species closely related to E. canis was reported to be infecting cattle and deer in British Columbia, Canada $[5,6]$. The isolation, in vitro culture, and molecular characterization of this agent was achieved later, when the same bacteria species was isolated from a partially engorged Rhipicephalus microplus female tick collected in Minas Gerais, Brazil [7,8]. In 2014, E. minasensis was isolated from dairy and beef cattle in Midwestern Brazil, and it was found to cause clinical ehrlichiosis in an experimentally infected calf [9].

In addition to R. microplus and other species of the genus Rhipicephalus, R. appendiculatus, R. eversti eversti, R. sanguineus s.l., and R. bursa [7,10,11], E. minasensis has also been identified in Amblyomma [11], Hyalomma [12,13], and Haemaphysalis [14], suggesting that several tick species could vector this bacterium. E. minasensis has a wide distribution; it has been reported in Canada [5,6], Brazil [7,9], Pakistan [13], Malaysia [15], China [14], Ethiopia [16], South Africa [11], and the Mediterranean island of Corsica [12]. These findings suggest that E. minasensis may be transmitted by more than one tick species which explain the wide geographical distribution of this bacterium [17].

In a previous report [9], the isolation of E. minasensis from a bovine blood sample and the in vitro culture of this bacterium in canine macrophage cell line DH82 were attempted. However, despite successful isolation, the propagation of the bacterium could not be established for more than one month [9]. In this study, we describe, for the first time, the isolation of E. minasensis from a blood sample of a naturally infected bovine. The successful propagation of E. minasensis was achieved in DH82 cells which were used for morphologic and genetic characterization of this bacterium by electron microscopy and genome sequencing, respectively. This study provides a comprehensive characterization of the E. minasensis strain Cuiabá and seeks to contribute to the better understanding of this pathogen.

\section{Material and Methods}

\subsection{Animal and Sample Collection}

An eight-month-old male calf of Holstein breed was admitted in May 2015 to the Veterinary Hospital of the Faculty of Veterinary Medicine of the Federal University of Mato Grosso State (UFMT). The calf belonged to a dairy herd infected with E. minasensis [9,10]. The farm is located at Santo Antônio do Leverger municipality, a small town $20 \mathrm{~km}$ distance from Cuiabá, the capital of Mato Grosso State, where practical classes of veterinary students of UFMT were carried out. A whole-blood sample was collected from the jugular vein in EDTA tubes, and hematological analyses were performed and compared with reference values previously reported [18].

\subsection{DNA Extraction and Polymerase Chain Reaction}

Genomic DNA was extracted from blood and DH82 cell culture, using the Wizard Genomic DNA purification Kit (Promega, Madison, WI, USA) according to the manufacturer's instructions. For molecular diagnosis, a heminested PCR protocol was used to amplify a fragment of the Ehrlichia gene $d s b$. The first PCR reaction targeted 401-bp (primers: Dsb-330 $5^{\prime}$ GATGATGTTTGAAGATATSAAACAAAT $3^{\prime}$ and Dsb-720 $5^{\prime}$ CTATTTTACTTCTTAAAGTTGATAWATC $3^{\prime}$ ), and the second reaction targeted 349-bp (primers: Dsb-380 5' ATTTTTAGRGATTTTCCAATACTTGG 3' and Dsb-720), as previously reported [9]. Amplification was carried out by following a protocol involving 1.25 U GoTaq ${ }^{\mathrm{TM}}$ Hot Start Polymerase (Promega, Madison, WI, USA) and $10 \mathrm{pmol} / \mu \mathrm{L}$ of each primer, according the manufacturer's instructions. DNA of E. canis strain Cuiabá\#1 and template free reactions were used as positive () and negative controls of the PCR reactions. Amplicons were visualized by agarose gel electrophoresis (1.5\%). 


\subsection{In Vitro Culture of Ehrlichia}

Blood samples were processed for isolation of Ehrlichia in cell culture. To this end, the blood was aseptically collected in EDTA vacuum tubes and transported to the laboratory for mononuclear cells (MNCs) isolation, using Histopaque 1083 (Sigma-Aldrich, St. Luis, MO, USA), as previously described [19]. Cultures were initiated by seeding the MNCs in a $25 \mathrm{~cm}^{2}$ culture flask containing Dulbecco's Modified Eagle's medium (DMEM, Sigma-Aldrich, St Louis, MO, USA) and supplemented with 20\% iron-fortified Bovine Calf Serum (BCS, Sigma-Aldrich, St Louis, MO, USA). The culture flask was kept at $37^{\circ} \mathrm{C}$ and $5 \% \mathrm{CO}_{2}$. Every two days, $1 / 5$ of the primary culture medium was collected for cytologic evaluation, using Romanowsky-stained smears (NewProv, Pinhais, PR, Brazil), and fresh medium was added. After 96 hours (h) of incubation, DH82 cells were added to the primary culture of MNCs, and the supplementation of DMEM was reduced to 5\% iron-fortified BCS. The culture was kept at $37^{\circ} \mathrm{C}$ and $5 \% \mathrm{CO}_{2}$. Every seven days, samples were collected for cytologic evaluation and PCR. Stocks of infected DH82 cells were resuspended in cell-freezing medium (Sigma-Aldrich, St Louis, MO, USA) and frozen at $-156^{\circ} \mathrm{C}$ in liquid nitrogen.

\subsection{Transmission Electron Microscopy}

The samples for transmission electron microscopy were prepared following protocols previously described [20]. Briefly, the monolayers of DH82 cells infected with E. minasensis were fixed in $2.5 \%$ paraformaldehyde and $0.1 \%$ glutaraldehyde in $0.05 \mathrm{M}$ cacodylate buffer, $\mathrm{pH} 7.3$, to which $0.01 \%$ trinitrophenol and $0.03 \% \mathrm{CaCl}_{2}$ were added. After fixation, the cells were washed with $0.1 \mathrm{M}$ cacodylate buffer, scraped off the flasks, and pelleted. The pellets were post-fixed in $1 \% \mathrm{OsO}_{4}$ in $0.1 \mathrm{M}$ of cacodylate buffer, en bloc stained with $2 \%$ aqueous uranyl acetate in $0.1 \mathrm{M}$ maleate buffer, dehydrated in ethanol, and embedded in Poly/Bed 812 (Polysciences, Warrington, PA, USA). Ultrathin sections were cut on Reichert-Leica Ultracut $S$ ultramicrotome, stained with lead citrate, and examined in a Philips 201 or CM-100 electron microscope at $60 \mathrm{kV}$.

\subsection{Genome Sequencing}

The genome of the E. minasensis strain Cuiabá was recently sequenced, annotated, and announced [21]. Briefly, DNA was extracted from DH82 cells infected with E. minasensis, and a Nextera XT library was prepared (Illumina, San Diego, CA, United States). Sequencing was performed using the sequencer NextSeq 550 System (Illumina, San Diego, United States). A total of 37 million reads passed the quality filter and were merged and assembled. Genome annotation was run with Prokka 1.14 [22]. The raw sequence reads were submitted to NCBI SRA and are available under the accession number PRJNA478569. The whole-genome shotgun project of the E. minasensis strain Cuiabá was deposited in DDBJ/EMBL/GenBank and is available under the accession number QOHL00000000. Genome assembly data are available under the accession number GCA_004181775.

\subsection{Core-Genome-Based Phylogenetic Tree}

Genome assemblies of E. canis (2 strains), E. chaffeensis (9 strains), E. muris (2 strains), E. ruminantium (10 strains), and E. minasensis (2 strains) available in the refseq database of NCBI were downloaded in fasta format. Genome accession numbers of the strains used are available in Supplementary Table S1. Three Anaplasma genomes (i.e., A. marginale-SAMN02603338, A. centrale-SAMN02604279 and A. phagocytophilum-SAMN02585077) were used as outgroups in the core-genome-based phylogenetic tree. The coding sequences of the genomes were used for ortholog genes identification, using the OrthoMCL pipeline [23]. The nucleotide sequences of all the ortholog genes identified in the 28 bacterial genomes were aligned using MACSE [24]. The final alignment included 97,908 nucleotide positions of 125 protein-coding genes present in the core-genome of the selected bacteria. A maximum-likelihood phylogenetic tree was then built by using the GTR model and the software PhyML 3.0 [25]. Reliability 
of internal branches was assessed by using the approximate likelihood ratio test (aLRT) implemented in Seaview [26].

\subsection{Bacterial Pan-Genome Profiles}

Gene clusters of Anaplasma and Ehrlichia genomes (see Section 2.6) were predicted with OrthoMCL [23], using best-matching gene similarities with an $E$-value $\leq 1 \times 10^{-5}$. The number of gene clusters was plotted against the number of genomes, and the resulting rarefaction curves were used to visualize the pan-genome and core-genome of Anaplasma and Ehrlichia. Rarefaction curves were built by using the settings recommended for datasets larger than 15 genomes. Pan-genome and core-genome profiles were calculated with the software PanGP [27].

\subsection{Genome Comparative Analysis}

The functional annotation of E. minasensis Cuiabá genome (SAMN09519635) was compared to that of other 9 genomes of the Order Rickettsiales: A. central Israel (SAMN02604279), A. marginale Florida-1 (SAMN02469629), A. phagocytophilum HZ2 (SAMN02604172), E. canis Jake (SAMN02598261), E. chaffeensis Arkansas (SAMN02604010), E. muris AS145 (SAMN02641637), E. ruminantium Welgevonden (SAMEA1705918), Rickettsia conorii Malish (SAMN02603141), and Rickettsia prowazekii Rp22 (SAMN02604077). All genomes were functionally annotated, using PATRIC [28], a web-based tool that uses RAST [29], which assigns gene functions and groups them according to different categories: superclass, class, subclass, and subsystems. The two-proportion test, implemented in R (prop.test function), was used to detect differences between the functional categories of E. minasensis and the other genomes, with a 95\% confidence interval [30].

\section{Results}

\subsection{Natural Infection of E. minasensis in a Calf}

An eight-month-old male calf of Holstein breed, from a dairy farm, presented fever, depression, and lethargy. The farm had a history of chronic R. microplus infestation and E. minasensis infection $[9,10]$. The animal was sent to the veterinary hospital, and routine examinations were performed by members of the faculty of veterinary medicine. Among the laboratory tests performed, the hematological analysis revealed abnormalities, including anemia $\left(4.7 \times 10^{6}\right.$ cells $/ \mathrm{mm}^{3}, 5.5 \mathrm{~g} / \mathrm{dl}$ hemoglobin, $18 \%$ packed cell volume, and 38 femtoliters (fl) of mean corpuscular volume), leukopenia $\left(3.7 \times 10^{3}\right.$ cells $\left./ \mathrm{mm}^{3}\right)$, and thrombocytopenia $\left(124 \times 10^{3}\right.$ platelets $\left./ \mathrm{mm}^{3}\right)$. Blood infection with Ehrlichia was confirmed by PCR amplification of a fragment of the Ehrlichia gene $d s b$.

\subsection{Isolation and In Vitro Culture of E. minasensis}

MNCs were isolated from the peripheral blood of the Ehrlichia-infected calf and seeded in DMEM supplemented with $20 \%$ BCS. After $72 \mathrm{~h}$ of incubation, irregular clusters of ehrlichial morulae were observed in monocytes of the primary culture (Figure 1).

One day after the ehrlichial morulae were observed in the primary culture, DH82 cells were added in the primary culture. Seven days later, ehrlichial morulae were observed within the cytoplasm of $10 \%$ of the DH82 cells (Figure 2). 


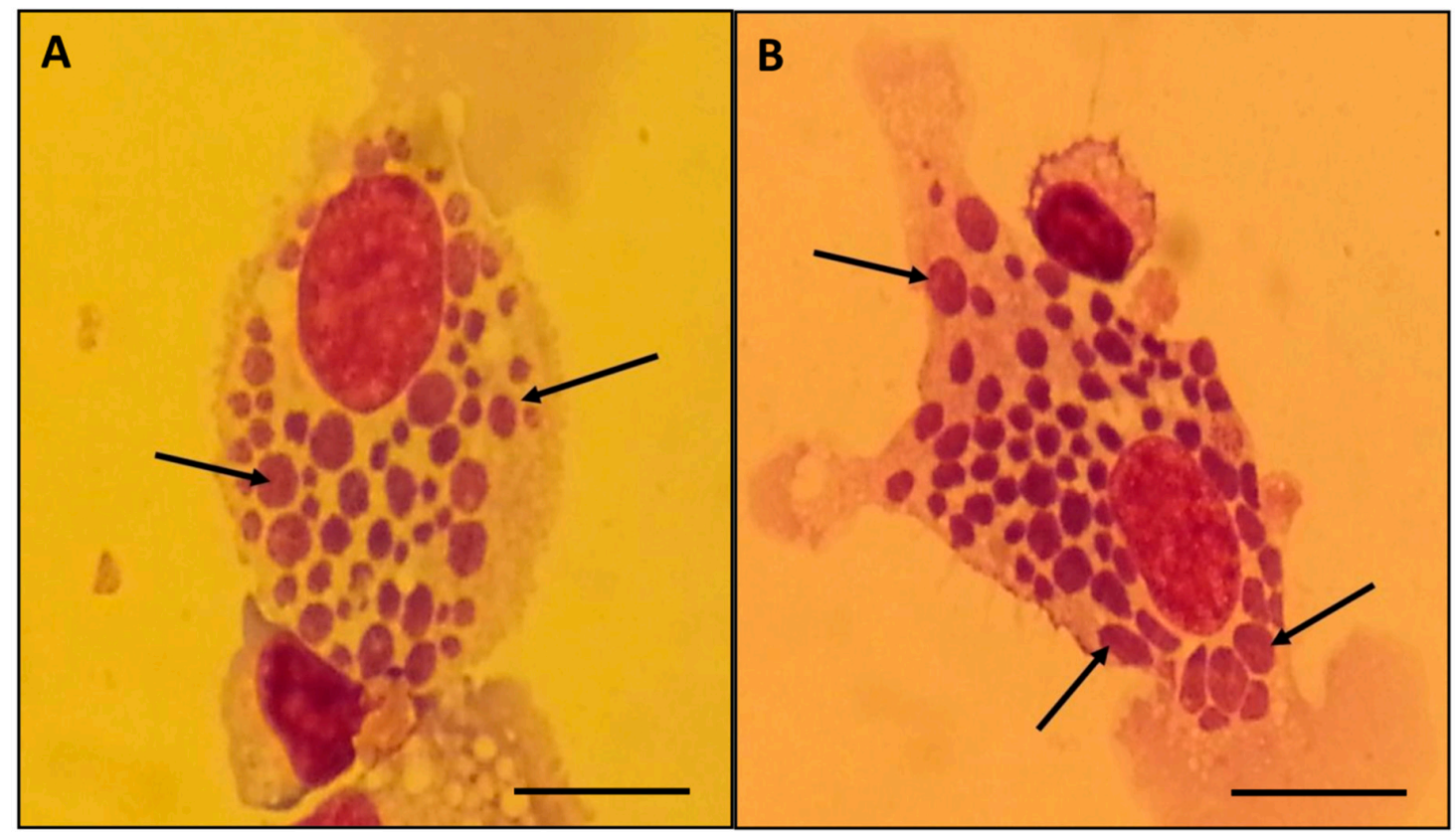

Figure 1. Romanowsky-stained smears showing large morulae of E. minasensis (arrows) in the cytoplasm of calf monocytes. Images (A) and (B) each show a different monocyte heavily infected with multiple morulae of E. minasensis. Magnification, $1000 \times$. Bar $=10 \mu \mathrm{m}$.

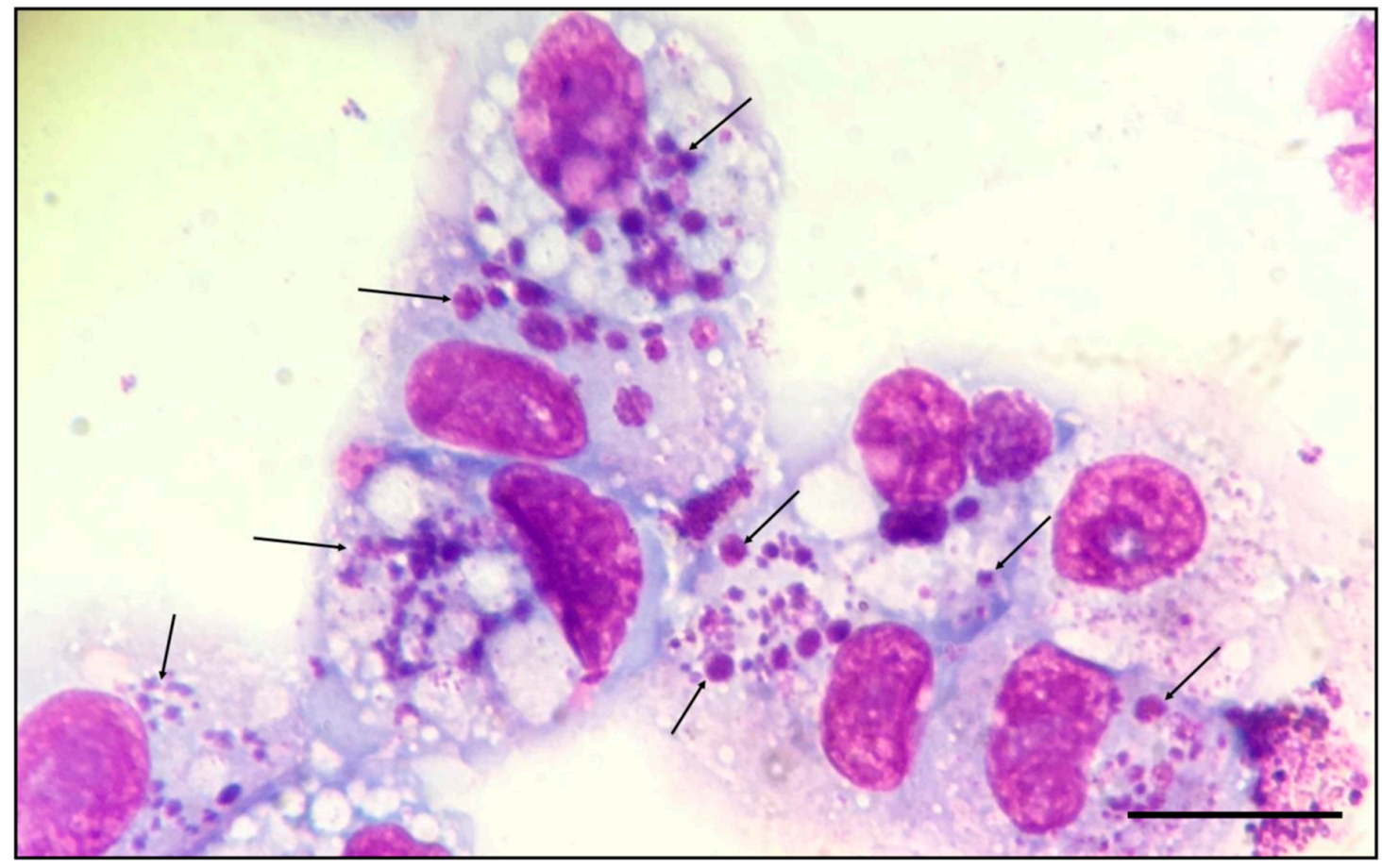

Figure 2. Romanowsky-stained stained smear showing morulae of E. minasensis (arrows) in the cytoplasm of DH82 cells. Magnification, 1000×. Bar $=10 \mu \mathrm{m}$.

After one month in culture, $90 \%-100 \%$ of DH82 cells were infected with E. minasensis. Subsequently, infected DH82 cells were subcultured into uninfected DH82 cells, and the infection reached $90 \%-100 \%$ within 10 to 14 days. Frozen stocks of Ehrlichia-infected DH82 cells were thawed, and their infectivity was tested. High infectivity was observed when the thawed material was added to uninfected DH82 monolayers. The E. minasensis isolate, designated as the Cuiabá strain, was deposited in the Ehrlichial 
collection of the Laboratory of Virology and Rickettsiosis of the Faculty of Veterinary Medicine of UFMT, where it is available upon request.

\subsection{Electron Microscopy Characterization}

E. minasensis developed within multiple parasitophorous vacuoles in the cytoplasm of DH82 cells (Figure 3A). The number of morulae per cell ranged from 1 to 16. The average number of microorganisms per morulae was five. Homogeneous populations of reticulate cells (RCs) (Figure 3B) and dense-cored cells (DCs) (Figure 3C) were observed.

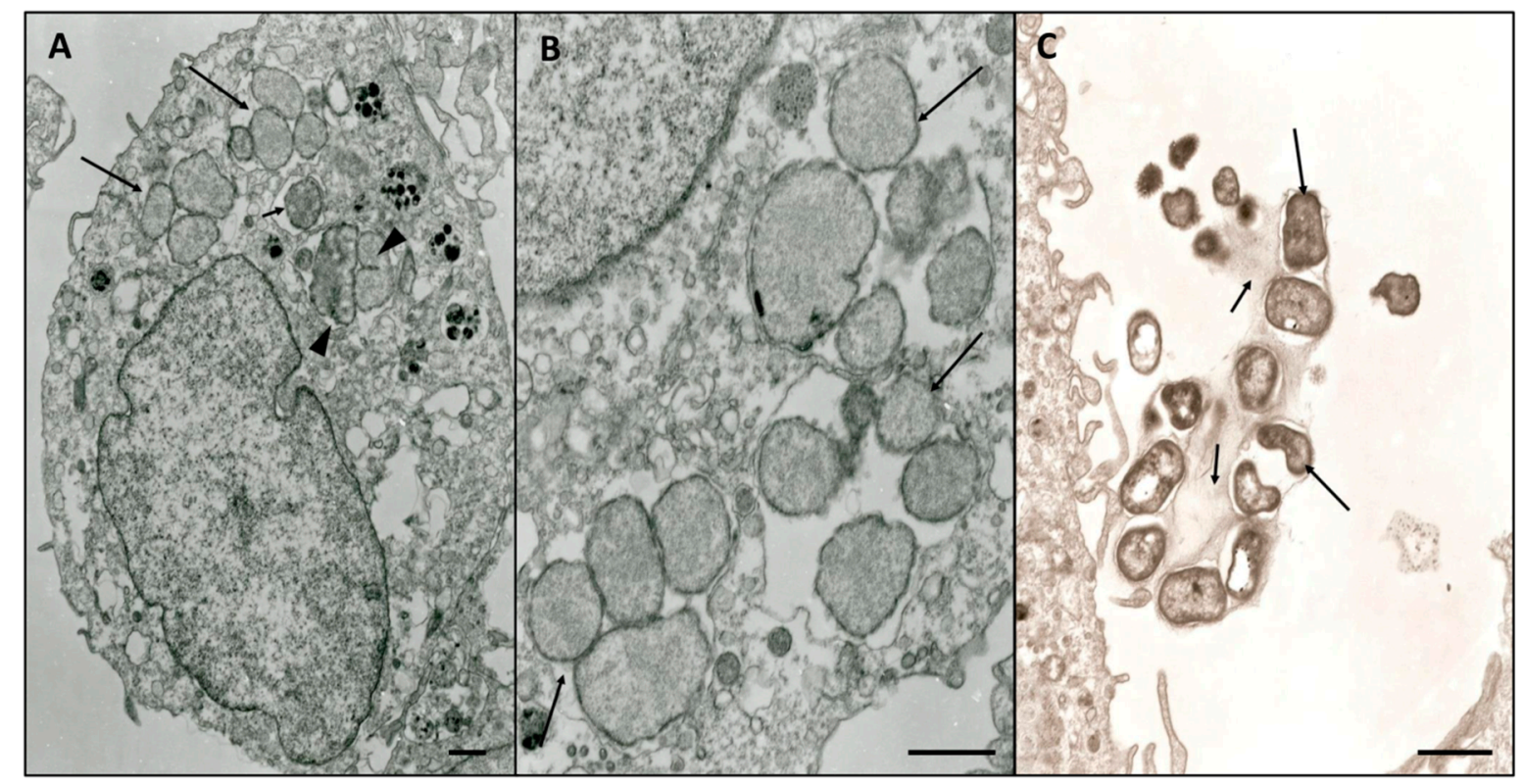

Figure 3. Electron micrograph of E. minasensis in DH82 cells. (A) Morulae were observed to contain reticulate cells (RC, large arrow), densely packed unique cell (small arrow), and cells dividing by unequal fission (head arrow). (B) RC bodies inside membrane-lined vacuoles were bound by an inner plasma membrane and an outer cell wall (arrow). (C) Dense-cored cells DC microorganisms (large arrow) surrounded by cellular plasmalemma (small arrow) after rupture of the host cell membrane. Bar $=1.0 \mu \mathrm{m}$.

RCs were small, oval and surrounded by two limiting membranes: an outer cell-wall membrane and inner cytoplasmic membrane. DCs were round with irregular or pleomorphic shape. Ehrlichial DCs were observed after the rupture of the host cell membrane. DCs were surrounded by the plasmalemma (Figure 3C). The diameter of the RCs ranged from 0.7 to $2.0 \times 0.6$ to $1.2 \mu \mathrm{m}$, while the DCs ranged from $0.5-1.5 \times 0.4$ to $1.0 \mu \mathrm{m}$.

\subsection{Genome Properties and Phylogenetic Analysis}

E. minasensis Cuiabá genome consists of 1,335,478bases, with an N50 of 694,769 and $29.5 \%$ G+C content, and revealed the presence of 1270 ORFs, including coding sequences (CDS, 1231) and RNA genes ( 3 rRNAs and 36 tRNAs). Comparison of general features of the E. minasensis Cuiabá genome with those of other Anaplasma, Ehrlichia, and Rickettsia species revealed similarities, but also differences between these genomes (Table 1).

Rarefaction curves built with gene clusters of 3 Anaplasma and 25 Ehrlichia genomes show that the pan-genome and core-genome of these bacteria have approximately 3000 and 400 genes, respectively (Supplementary Figure S1). A maximum-likelihood phylogenetic tree was built based on the alignment of 97,908 nucleotide positions of 125 CDS of the core-genome of Anaplasma, Ehrlichia, and Rickettsia (Figure 4). 
Table 1. Genome properties.

\begin{tabular}{ccccccccccc}
\hline \multirow{2}{*}{ Features * } & \multicolumn{10}{c}{ Bacteria } \\
\cline { 2 - 11 } & EMI & ECA & ECH & EMU & ER & AC & AM & AP & RC & RP \\
\hline Size (bp) & $1,335,478$ & $1,315,030$ & $1,176,248$ & $1,196,717$ & $1,516,355$ & $1,206,806$ & $1,136,981$ & $1,477,581$ & $1,268,755$ & $1,111,612$ \\
GC (\%) & 29.5 & 29.0 & 30.1 & 29.7 & 27.5 & 50.0 & 49.8 & 42.0 & 32.4 & 29.0 \\
tRNA & 36 & 36 & 37 & 37 & 36 & 37 & 35 & 37 & 33 & 33 \\
rRNA & 3 & 2 & 2 & 2 & 2 & 2 & 3 & 3 & 2 & 2 \\
total ORFs & 1270 & 1068 & 1032 & 1055 & 1056 & 1194 & 1359 & 1501 & 1637 & 960 \\
PATRIC CDS & 1231 & 1030 & 992 & 992 & 1015 & 1153 & 1321 & 1447 & 1578 & 919 \\
Contigs & 55 & 1 & 1 & 1 & 1 & 1 & 204 & 1 & 1 & 1 \\
\hline
\end{tabular}

* Features based on PATRIC annotations of one strain of each species. Abbreviations as follow: EMI, E. minasensis Cuiabá; ECA, E. canis; ECH, E. chaffeensis; EMU, E. muris; ER, E. ruminantium; AC, A. centrale; AM, A. marginale; $\mathrm{AP}, A$. phagocytophilum; RC, R. conorii; RP, R. prowazekii. Genome accessions are available in material and methods (Section 2.8).

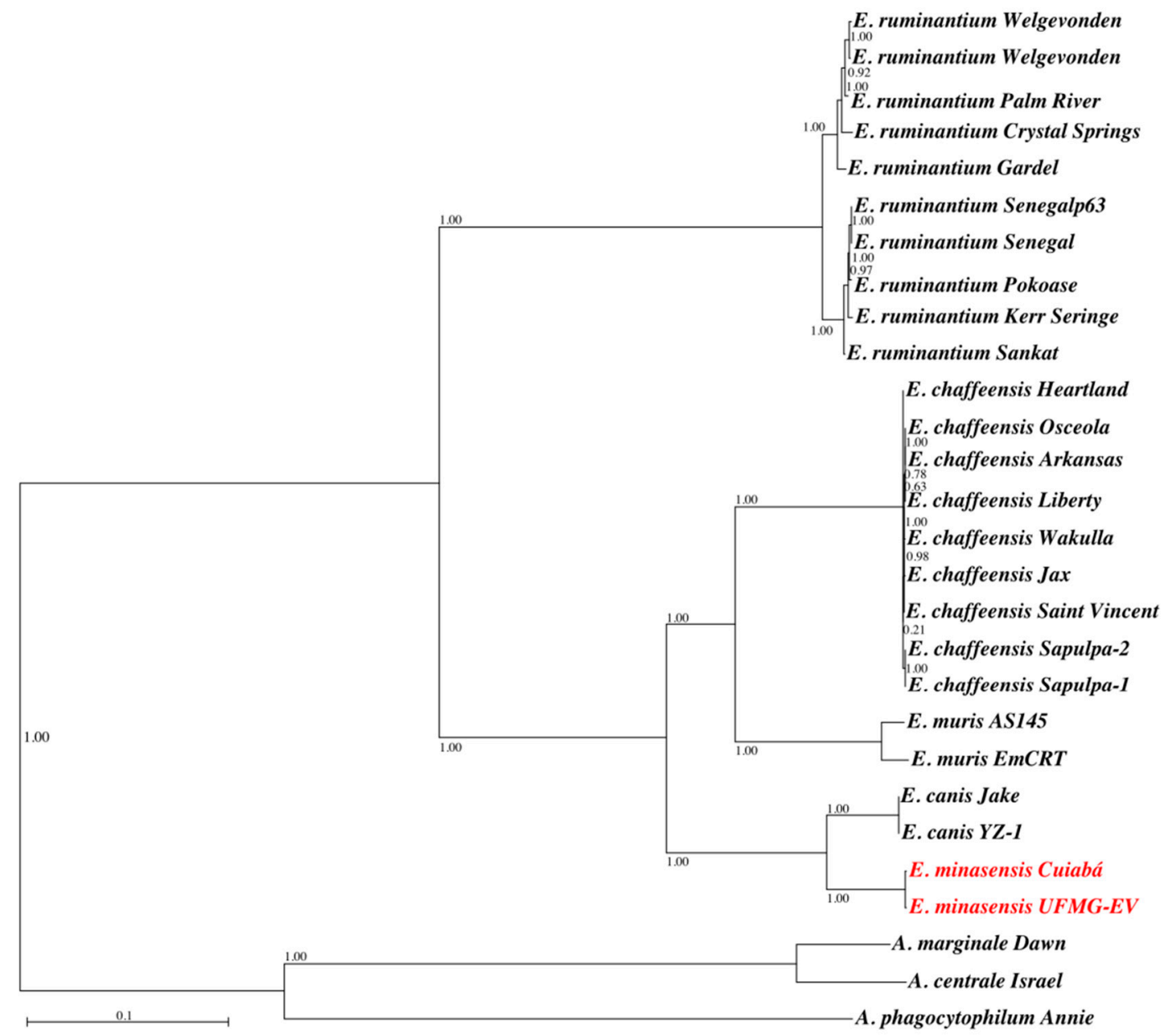

Figure 4. Core-genome-based phylogenetic tree of Ehrlichia. A maximum-likelihood phylogenetic tree was built, using the GTR model and the software PhyML. The final alignment included the nucleotide sequences of 125 protein-coding genes present in the core-genome of the selected bacteria. The genomes of $A$. marginale, A. centrale, and A. phagocytophilum were included as outgroups. The two strains of $E$. minasensis are highlighted in red. Values on internal branches represent the statistical support of the topology calculated by aLRT.

The phylogenetic tree shows that E. minasensis strains Cuiabá and UFMG-EV cluster together and are both closely related to E. canis (Figure 4). 


\subsection{Genome Comparison Analysis}

Genome functional annotations of E. minasensis strain Cuiabá were compared to those of other species of Anaplasma, Ehrlichia, and Rickettsia (Table 2).

Table 2. Comparison of genome functional features.

\begin{tabular}{|c|c|c|c|c|c|c|c|c|c|c|}
\hline Class & EMI & ECA & ECH & EMU & ER & AC & AM & AP & RC & $\mathbf{R P}$ \\
\hline $\begin{array}{l}\text { Amino Acids and } \\
\text { Derivatives }\end{array}$ & $\begin{array}{c}0.034 \\
(42)\end{array}$ & $\begin{array}{c}0.036 \\
(37)\end{array}$ & $\begin{array}{c}0.037 \\
(37)\end{array}$ & $\begin{array}{c}0.041 \\
(41)\end{array}$ & $\begin{array}{c}0.042 \\
(43)\end{array}$ & $\begin{array}{c}0.021 \\
(24)\end{array}$ & $\begin{array}{c}0.030 \\
(39)\end{array}$ & $\begin{array}{l}0.005^{* * *} \\
(7)\end{array}$ & $\begin{array}{l}0.015^{* *} \\
(24)\end{array}$ & $\begin{array}{c}0.026 \\
(24)\end{array}$ \\
\hline Carbohydrates & $\begin{array}{c}0.002 \\
(2)\end{array}$ & $\begin{array}{l}0.002 \\
(2)\end{array}$ & $\begin{array}{c}0.002 \\
(2)\end{array}$ & $\begin{array}{l}0.002 \\
(2)\end{array}$ & $\begin{array}{l}0.002 \\
(2)\end{array}$ & $\begin{array}{l}0.002 \\
(2)\end{array}$ & $\begin{array}{c}0.002 \\
(2)\end{array}$ & $\begin{array}{c}0.001 \\
(2)\end{array}$ & $\begin{array}{l}0.001 \\
(2)\end{array}$ & $\begin{array}{c}0.002 \\
(2)\end{array}$ \\
\hline $\begin{array}{l}\text { Cell Cycle, Cell Division } \\
\text { and Death }\end{array}$ & $\begin{array}{c}0.011 \\
(13)\end{array}$ & $\begin{array}{l}0.006 \\
(6)\end{array}$ & $\begin{array}{l}0.006 \\
(6)\end{array}$ & $\begin{array}{l}0.006 \\
(6)\end{array}$ & $\begin{array}{l}0.006 \\
(6)\end{array}$ & $\begin{array}{l}0.029^{* *} \\
(33)\end{array}$ & $\begin{array}{l}0.036^{* * *} \\
(47)\end{array}$ & $\begin{array}{c}0.004 \\
(6)\end{array}$ & $\begin{array}{l}0.029^{* *} \\
(45)\end{array}$ & $\begin{array}{l}0.032^{* * *} \\
(29)\end{array}$ \\
\hline $\begin{array}{l}\text { Cell Envelope, Capsule } \\
\text { and Slime layer }\end{array}$ & $\begin{array}{c}0.002 \\
(2)\end{array}$ & $\begin{array}{c}0.002 \\
(2)\end{array}$ & $\begin{array}{l}0.002 \\
(2)\end{array}$ & $\begin{array}{c}0.002 \\
(2)\end{array}$ & $\begin{array}{c}0.002 \\
(2)\end{array}$ & $\begin{array}{l}0.004 \\
(5)\end{array}$ & $\begin{array}{l}0.004 \\
(5)\end{array}$ & $\begin{array}{c}0.001 \\
(2)\end{array}$ & $\begin{array}{l}0.008^{*} \\
(13)\end{array}$ & $\begin{array}{c}0.014^{* *} \\
(13)\end{array}$ \\
\hline $\begin{array}{l}\text { Clustering-based } \\
\text { subsystems }\end{array}$ & $\begin{array}{c}0.003 \\
(4)\end{array}$ & $\begin{array}{l}0.0165^{* *} \\
(17)\end{array}$ & $\begin{array}{c}0.017^{* *} \\
(17)\end{array}$ & $\begin{array}{c}0.016^{* *} \\
(16)\end{array}$ & $\begin{array}{l}0.016^{* *} \\
(16)\end{array}$ & $\begin{array}{l}0.015^{* *} \\
(17)\end{array}$ & $\begin{array}{c}0.004 \\
(5)\end{array}$ & $\begin{array}{c}0.007 \\
(10)\end{array}$ & $\begin{array}{c}0.003 \\
(4)\end{array}$ & $\begin{array}{c}0.004 \\
(4)\end{array}$ \\
\hline $\begin{array}{l}\text { Cofactors, Vitamins, } \\
\text { Prosthetic Groups }\end{array}$ & $\begin{array}{l}0.084 \\
(104)\end{array}$ & $\begin{array}{l}0.098 \\
(101)\end{array}$ & $\begin{array}{l}0.102 \\
(101)\end{array}$ & $\begin{array}{l}0.104 \\
(103)\end{array}$ & $\begin{array}{l}0.101 \\
(103)\end{array}$ & $\begin{array}{l}0.087 \\
(100)\end{array}$ & $\begin{array}{l}0.090 \\
(119)\end{array}$ & $\begin{array}{l}0.077 \\
(111)\end{array}$ & $\begin{array}{l}0.042^{* * *} \\
(66)\end{array}$ & $\begin{array}{c}0.069 \\
(63)\end{array}$ \\
\hline DNA Processing & $\begin{array}{c}0.032 \\
(40)\end{array}$ & $\begin{array}{c}0.039 \\
(40)\end{array}$ & $\begin{array}{c}0.040 \\
(40)\end{array}$ & $\begin{array}{c}0.042 \\
(42)\end{array}$ & $\begin{array}{c}0.044 \\
(45)\end{array}$ & $\begin{array}{c}0.042 \\
(49)\end{array}$ & $\begin{array}{c}0.048 \\
(63)\end{array}$ & $\begin{array}{c}0.036 \\
(52)\end{array}$ & $\begin{array}{c}0.034 \\
(54)\end{array}$ & $\begin{array}{c}0.038 \\
(35)\end{array}$ \\
\hline $\begin{array}{l}\text { Energy and Precursor } \\
\text { Metabolites Generation }\end{array}$ & $\begin{array}{c}0.037 \\
(46)\end{array}$ & $\begin{array}{c}0.045 \\
(46)\end{array}$ & $\begin{array}{l}0.046 \\
(46)\end{array}$ & $\begin{array}{c}0.050 \\
(50)\end{array}$ & $\begin{array}{c}0.046 \\
(47)\end{array}$ & $\begin{array}{c}0.040 \\
(46)\end{array}$ & $\begin{array}{c}0.039 \\
(52)\end{array}$ & $\begin{array}{l}0.037 \\
(53)\end{array}$ & $\begin{array}{c}0.027 \\
(42)\end{array}$ & $\begin{array}{c}0.041 \\
(38)\end{array}$ \\
\hline $\begin{array}{l}\text { Fatty Acids, Lipids, and } \\
\text { Isoprenoids }\end{array}$ & $\begin{array}{c}0.037 \\
(45)\end{array}$ & $\begin{array}{c}0.043 \\
(44)\end{array}$ & $\begin{array}{c}0.044 \\
(44)\end{array}$ & $\begin{array}{c}0.045 \\
(45)\end{array}$ & $\begin{array}{c}0.041 \\
(42)\end{array}$ & $\begin{array}{c}0.036 \\
(42)\end{array}$ & $\begin{array}{c}0.028 \\
(37)\end{array}$ & $\begin{array}{c}0.029 \\
(42)\end{array}$ & $\begin{array}{l}0.018^{* *} \\
(28)\end{array}$ & $\begin{array}{c}0.030 \\
(28)\end{array}$ \\
\hline Membrane Transport & $\begin{array}{c}0.044 \\
(54)\end{array}$ & $\begin{array}{c}0.046 \\
(47)\end{array}$ & $\begin{array}{c}0.047 \\
(47)\end{array}$ & $\begin{array}{c}0.049 \\
(49)\end{array}$ & $\begin{array}{c}0.054 \\
(55)\end{array}$ & $\begin{array}{c}0.056 \\
(65)\end{array}$ & $\begin{array}{c}0.058 \\
(77)\end{array}$ & $\begin{array}{c}0.039 \\
(57)\end{array}$ & $\begin{array}{c}0.037 \\
(59)\end{array}$ & $\begin{array}{c}0.063 \\
(58) \\
\end{array}$ \\
\hline $\begin{array}{l}\text { Metabolite damage and } \\
\text { its repair or mitigation }\end{array}$ & $\begin{array}{c}0.003 \\
(4)\end{array}$ & $\begin{array}{c}0.004 \\
(4)\end{array}$ & $\begin{array}{c}0.004 \\
(4)\end{array}$ & $\begin{array}{c}0.004 \\
(4)\end{array}$ & $\begin{array}{c}0.004 \\
(4)\end{array}$ & $\begin{array}{c}0.003 \\
(4)\end{array}$ & $\begin{array}{c}0.003 \\
(4)\end{array}$ & $\begin{array}{c}0.003 \\
(4)\end{array}$ & $\begin{array}{c}0.003 \\
(4)\end{array}$ & $\begin{array}{c}0.003 \\
(3)\end{array}$ \\
\hline Miscellaneous & $\begin{array}{l}0.001 \\
(1)\end{array}$ & $\begin{array}{c}0.001 \\
(1)\end{array}$ & $\begin{array}{c}0.001 \\
(1)\end{array}$ & $\begin{array}{l}0.001 \\
(1)\end{array}$ & $\begin{array}{c}0.001 \\
(1)\end{array}$ & $\begin{array}{c}0.002 \\
(2)\end{array}$ & $\begin{array}{c}0.002 \\
(2)\end{array}$ & $\begin{array}{c}0.001 \\
(1)\end{array}$ & $\begin{array}{c}0.000 \\
(0)\end{array}$ & $\begin{array}{c}0.001 \\
(1)\end{array}$ \\
\hline $\begin{array}{l}\text { Nucleosides and } \\
\text { Nucleotides }\end{array}$ & $\begin{array}{c}0.025 \\
(31)\end{array}$ & $\begin{array}{c}0.030 \\
(31)\end{array}$ & $\begin{array}{c}0.031 \\
(31)\end{array}$ & $\begin{array}{c}0.033 \\
(33)\end{array}$ & $\begin{array}{c}0.031 \\
(31)\end{array}$ & $\begin{array}{c}0.027 \\
(31)\end{array}$ & $\begin{array}{c}0.026 \\
(35)\end{array}$ & $\begin{array}{c}0.029 \\
(42)\end{array}$ & $\begin{array}{l}0.001^{* * *} \\
(2)\end{array}$ & $\begin{array}{c}0.002 \\
(2)\end{array}$ \\
\hline Phosphate Metabolism & $\begin{array}{c}0.003 \\
(4)\end{array}$ & $\begin{array}{c}0.004 \\
(4)\end{array}$ & $\begin{array}{c}0.004 \\
(4) \\
\end{array}$ & $\begin{array}{c}0.004 \\
(4) \\
\end{array}$ & $\begin{array}{c}0.004 \\
(4)\end{array}$ & $\begin{array}{c}0.003 \\
(4)\end{array}$ & $\begin{array}{c}0.005 \\
(7)\end{array}$ & $\begin{array}{c}0.003 \\
(4)\end{array}$ & $\begin{array}{c}0.000 \\
(0)\end{array}$ & $\begin{array}{c}0.000 \\
(0)\end{array}$ \\
\hline $\begin{array}{c}\text { Prokaryotic cell type } \\
\text { differentiation }\end{array}$ & $\begin{array}{c}0.001 \\
(1)\end{array}$ & $\begin{array}{l}0.001 \\
(1)\end{array}$ & $\begin{array}{c}0.001 \\
(1)\end{array}$ & $\begin{array}{l}0.001 \\
(1)\end{array}$ & $\begin{array}{c}0.001 \\
(1)\end{array}$ & $\begin{array}{c}0.001 \\
(1)\end{array}$ & $\begin{array}{c}0.001 \\
(1)\end{array}$ & $\begin{array}{c}0.001 \\
(1)\end{array}$ & $\begin{array}{c}0.001 \\
(1)\end{array}$ & $\begin{array}{c}0.001 \\
(1)\end{array}$ \\
\hline $\begin{array}{l}\text { Protein Fate (folding, } \\
\text { modification, targeting, } \\
\text { degradation) }\end{array}$ & $\begin{array}{c}0.024 \\
(29)\end{array}$ & $\begin{array}{c}0.028 \\
(29)\end{array}$ & $\begin{array}{c}0.029 \\
(29)\end{array}$ & $\begin{array}{c}0.029 \\
(29)\end{array}$ & $\begin{array}{c}0.029 \\
(29)\end{array}$ & $\begin{array}{c}0.024 \\
(28)\end{array}$ & $\begin{array}{c}0.024 \\
(32)\end{array}$ & $\begin{array}{c}0.020 \\
(29)\end{array}$ & $\begin{array}{c}0.016 \\
(26)\end{array}$ & $\begin{array}{c}0.028 \\
(26)\end{array}$ \\
\hline Protein Synthesis & $\begin{array}{l}0.130 \\
(160)\end{array}$ & $\begin{array}{l}0.149 \\
(153)\end{array}$ & $\begin{array}{l}0.154 \\
(153)\end{array}$ & $\begin{array}{l}0.119 \\
(118)\end{array}$ & $\begin{array}{l}0.157 \\
(159)\end{array}$ & $\begin{array}{c}0.101^{*} \\
(117)\end{array}$ & $\begin{array}{l}0.129 \\
(170)\end{array}$ & $\begin{array}{l}0.112 \\
(162)\end{array}$ & $\begin{array}{c}0.097^{* *} \\
(153)\end{array}$ & $\begin{array}{c}0.164^{*} \\
(151)\end{array}$ \\
\hline Respiration & $\begin{array}{c}0.076 \\
(94)\end{array}$ & $\begin{array}{c}0.089 \\
(92)\end{array}$ & $\begin{array}{c}0.093 \\
(92)\end{array}$ & $\begin{array}{c}0.098 \\
(97)\end{array}$ & $\begin{array}{l}0.101^{*} \\
(103)\end{array}$ & $\begin{array}{l}0.075^{* * *} \\
(87)\end{array}$ & $\begin{array}{c}0.074 \\
(98)\end{array}$ & $\begin{array}{c}0.068 \\
(99)\end{array}$ & $\begin{array}{c}0.059 \\
(93)\end{array}$ & $\begin{array}{l}0.108^{*} \\
(99)\end{array}$ \\
\hline RNA Processing & $\begin{array}{c}0.030 \\
(37)\end{array}$ & $\begin{array}{c}0.028 \\
(29)\end{array}$ & $\begin{array}{c}0.029 \\
(29)\end{array}$ & $\begin{array}{c}0.034 \\
(34)\end{array}$ & $\begin{array}{c}0.031 \\
(31)\end{array}$ & $\begin{array}{c}0.025 \\
(29)\end{array}$ & $\begin{array}{c}0.030 \\
(40)\end{array}$ & $\begin{array}{c}0.020 \\
(29)\end{array}$ & $\begin{array}{c}0.032 \\
(50)\end{array}$ & $\begin{array}{c}0.051^{*} \\
(47)\end{array}$ \\
\hline $\begin{array}{l}\text { Stress Response, Defense } \\
\text { and Virulence }\end{array}$ & $\begin{array}{c}0.040 \\
(49)\end{array}$ & $\begin{array}{c}0.038 \\
(39)\end{array}$ & $\begin{array}{c}0.039 \\
(39)\end{array}$ & $\begin{array}{c}0.040 \\
(40)\end{array}$ & $\begin{array}{c}0.040 \\
(41)\end{array}$ & $\begin{array}{c}0.035 \\
(40)\end{array}$ & $\begin{array}{c}0.042 \\
(56)\end{array}$ & $\begin{array}{l}0.025^{*} \\
(36)\end{array}$ & $\begin{array}{l}0.034 \\
(54)\end{array}$ & $\begin{array}{c}0.049 \\
(45)\end{array}$ \\
\hline
\end{tabular}

Comparison of genes per class, according to PATRIC classification. Values correspond with the proportion of each class based on the total CDS identified. Number of CDS within each class are inside brackets. Significant differences with regard to E. minasensis Cuiabá are indicated by ${ }^{*}(p<0.05),{ }^{* *}(p<0.01)$, and ${ }^{* * *}(p<0.001)$. Abbreviations are as follow: EMI, E. minasensis; ECA, E. canis; ECH, E. chaffeensis; EMU, E. muris; ER, E. ruminantium; AC, A. centrale; $\mathrm{AM}, A$. marginale; AP, A. phagocytophilum; RC, R. conorii; and RP, R. prowazekii. Genome accessions are available in material and methods (Section 2.8).

Major functional categories were distributed across 20 classes, and no significant differences were found in the amount of genes involved in carbohydrate metabolism, DNA processing, energy and precursor metabolites generation, membrane transport, metabolite damage and its repair or mitigation, phosphate metabolism, prokaryotic cell type differentiation, protein fate (i.e., folding, 
modification, targeting, and degradation), and miscellaneous. This suggests that the amount of functions in these categories is highly conserved among the selected Rickettsiales. For the rest of the 11 categories, differences were found between E. minasensis and at least one of the other bacteria. The only significant difference between E. minasensis and E. canis, E. chaffeensis, and E. muris was in the category clustering-based subsystems in which E. minasensis showed a significantly lower number of genes (i.e., four) compared with these three Ehrlichia (i.e., between 16 and 17). A pairwise comparison between E. minasensis and E. ruminantium revealed that E. minasensis has a significant reduction in genes of clustering-based subsystems (i.e., 16 in E. ruminantium and four in E. minasensis) and respiration (i.e., 103 in E. ruminantium and 94 in E. minasensis). Concerning Anaplasma and Rickettsia, E. minasensis genome functional annotation had significant differences with $A$. centrale (four categories), A. marginale (one category), A. phagocytophilum (two categories), R. conorii (seven categories), and R. prowazekii (five categories) (Table 2).

\section{Discussion}

This paper reports the isolation of $E$. minasensis from a naturally infected calf from the midwestern region of Brazil. E. minasensis was previously isolated from R. microplus ticks [2,7], and it was already known that this bacterium was able to infect cattle and, after experimental infection, produce clinical manifestations associated with ehrlichiosis [9]. However, this is the first report of an E. minasensis strain isolated directly from a naturally infected animal with clinical signs of ehrlichiosis. In addition to E. minasensis, the other Ehrlichia species that infects ruminants is E. ruminantium, which is the causative agent of heartwater or cowdriosis mainly in African bovines [3]. While E. ruminantium is a recognized cause of ehrlichiosis in bovines, the ability of $E$. minasensis to cause this disease has been overlooked [17].

The clinical signs and blood parameters of the naturally infected calf described in this study concurred with those previously described for a calf experimentally infected with E. minasensis [9]. The clinical signs caused by virulent $E$. ruminantium strains include elevated temperature, loss of appetite, heavy breathing, hanging head, depression, exaggerated blinking and chewing movements, anorexia, hyperesthesia, lacrimation, convulsions, and death [3]. As most of the clinical signs produced by E. minasensis and E. ruminantium would not individually constitute a definitive diagnosis, and there is also an overlap of some signs (e.g., fever and depression), the postmortem examination plays a crucial role in the differential diagnosis of these two Ehrlichia. As the macroscopic lesions produced by E. ruminantium are different to those produced by E. minasensis, a differential postmortem diagnosis can be established for the infection with these pathogens. Common lesions caused by E. ruminantium include hydropericardium, hydrothorax, and increased vascular permeability, which are associated with edema of the lungs, brain, and other organs [3]. In contrast, the major macroscopic lesion observed in animals experimentally infected with E. minasensis was enlargement and diffuse swelling of mesenteric lymph nodes, and no pathologic changes were observed in the lung, spleen, kidney, stomach, and brain [9]. Histopathological examination is also useful, as E. ruminantium morulae are found in the cytoplasm of endothelial cells, whereas E. minasensis morulae are found mostly in the cytoplasm of MNCs of peripheral blood. The clinical signs caused by E. minasensis in bovines appear more similar to those of canine ehrlichiosis caused by E. canis in dogs [9,31], in which a chronic development of the disease with an asymptomatic phase is typical [32]. Experimental infection with E. minasensis was also reported in splenectomized calves, using the BC strain in Canada; however no clinical manifestations were observed, probably due to the use of a nonpathogenic $E$. minasensis strain $[5,33]$.

In order to ensure the transfer of the microorganisms from the host MNCs to the cell line DH82, two major procedures were followed. First, the primary culture of bovine MNCs was incubated for $96 \mathrm{~h}$, in order to increase the infection rates of Ehrlichia. Second, after the visualization of a large number of Ehrlichia morulae, uninfected DH82 cells were added to the primary culture. This strategy enabled the isolation of E. minasensis into DH82 cells. Earlier isolation of E. minasensis was achieved 
successfully in IDE8 cells, which were used as the source of infection for the DH82 cells [8]. In this study, it was possible to isolate E. minasensis in DH82 cells directly from infected bovine cells.

Ultrastructural characterization of E. minasensis Cuiabá showed that this isolate has typical morphological features of the genus Ehrlichia. Among these features are the presence of pleomorphic RC and DC cells surrounded by two membranes (the cell membrane and cytoplasmic membrane), the location of microorganisms within membrane-bound compartments (morulae), and the size of the cells which are comparable with those of other Ehrlichia cultured in mammalian cells [20,34]. In one of the images (Figure 3C), it was possible to observe the DC cells leaving the host cell and moving to the extracellular environment, after the rupture of the morulae.

In agreement with previous molecular analysis using the bacterial genes $16 \mathrm{~S}$ rRNA, groEL, $d s b, g l t A$, and trp36 [7,9], the core-genome-based phylogenetic tree confirmed that E. minasensis is a sister taxa of the dog pathogen E. canis, and, despite infecting the same host, E. minasensis and E. ruminantium are distantly related pathogens [2]. Previous molecular evolution analysis based in the gene trp36 suggested that E. minasensis evolved from highly variable strains of E. canis [35]. Genome comparison analysis showed that the E. minasensis Cuiabá genome is bigger and has more CDS than that of E. canis. This suggests that diversification and evolution of host-shift (i.e., from dogs to cattle, [35]) in E. minasensis might had been related to genome expansion and the acquisition of new genes associated with virulence and infection in cattle. The genome expansion could also be explained by an increase in the junk DNA sequence. However, the discrimination between the factors explaining the genome expansion in E. minasensis, compared with E. canis, is out of the scope of this manuscript. A high-resolution comparison of the genomes of E. canis and E. minasensis will contribute to understanding the host-shift in Ehrlichia. The rationale behind this idea is that pathogen adaptation to new hosts is usually associated with changes in the genome [36]. Indeed, recent studies have investigated host-shifts, with an emphasis on genetic changes, such as mutations, hybridizations, chromosomal reorganizations, or horizontal gene transfer events, involved in host-shift genetics [36]. Host-shift can involve multiple factors; and certainly, other factors influencing host-shift such as phenotypic plasticity and/or cryptic genetic variation in the pathogen population should also be considered. In this preliminary study, the only functional category found to be significantly different between E. minasensis Cuiabá and E. canis was clustering-based subsystems in which E. minasensis has significantly less genes than E. canis and the other Ehrlichia. Clustering-based subsystems include functionally coupled genes (genes found proximal to each other in the genomes of diverse taxa) for which the functional attributes are not well understood. However, it is noteworthy that, despite the fact that the differences were not significant, E. minasensis has more genes than E. canis, in other important categories, such as membrane transport; cell cycle, division, and death; protein synthesis; RNA processing; and stress response, defense, and virulence. It is also important to mention that a single gene could be functionally relevant and provide an adaptive advantage in new environments.

\section{Conclusions}

The isolation and characterization of E. minasensis Cuiabá from a naturally infected bovine, together with the previous report of experimental infection of this bacterium in the same type of host [9], confirms that E. minasensis is a novel pathogen that causes bovine ehrlichiosis in Brazil. However, further studies are required to elucidate the potential pathogenicity of $E$. minasensis strains found in countries other than Brazil. In addition to adding genetic information to the study of the Anaplasmataceae family, the access to the E. minasensis genome will help elucidating the genetic basis of host-shift in Ehrlichia. The identification of major antigenic proteins of E. minasensis will be a crucial step towards the development of diagnostic tests and vaccines to control infection by thispathogen. 
Supplementary Materials: Supplementary materials can be found at http:/www.mdpi.com/2076-2607/7/11/528/s1.

Author Contributions: D.M.d.A. and A.C.-C. conceived the study, drafted the manuscript and analyzed data. J.P.A.J. prepared Nextera XT and genome sequencing, L.N. performed the genome annotation. E.M.C. attended the clinical case at the veterinary hospital. L.A.-B., F.V. and E.B. performed genome analyses. V.L.P. performed electron microscopy analysis and interpretation of morphological data. All authors contributed to the preparation of the final version of the manuscript.

Funding: The authors gratefully acknowledge the National Council for Scientific and Technological Development (CNPq) for the financial support (process no. 443923/2014-0) and for awarding a research-productivity grant to D.M. Aguiar, J.P. Araujo Jr, and L. Nakazato. The first author thanks God for his life and for his work in veterinary medicine.

Conflicts of Interest: The authors declare no conflict of interest.

\section{References}

1. Dumler, J.S.; Barbet, A.F.; Bekker, C.P.; Dasch, G.A.; Palmer, G.H.; Ray, S.C.; Rikihisa, Y.; Rurangirwa, F.R. Reorganization of genera in the families Rickettsiaceae and Anaplasmataceae in the order Rickettsiales: Unification of some species of Ehrlichia with Anaplasma, Cowdria with Ehrlichia and Ehrlichia with Neorickettsia, descriptions of six new species combinations and designation of Ehrlichia equi and 'HGE agent' as subjective synonyms of Ehrlichia phagocytophila. Int. J. Syst. Evol. Microbiol. 2001, 51, 2145-2165. [CrossRef] [PubMed]

2. Cabezas-Cruz, A.; Zweygarth, E.; Vancová, M.; Broniszewska, M.; Grubhoffer, L.; Passos, L.M.F.; Ribeiro, M.F.B.; Alberdi, P.; de la Fuente, J. Ehrlichia minasensis sp. nov., isolated from the tick Rhipicephalus microplus. Int. J. Syst. Evol. Microbiol. 2016, 66, 1426-1430. [CrossRef] [PubMed]

3. Allsopp, B.A. Heartwater-Ehrlichia ruminantium infection. Rev. Sci. Tech. 2015, 34, 557-568. [CrossRef] [PubMed]

4. Gondard, M.; Cabezas-Cruz, A.; Charles, R.A.; Vayssier-Taussat, M.; Albina, E.; Moutailler, S. Ticks and Tick-Borne Pathogens of the Caribbean: Current Understanding and Future Directions for More Comprehensive Surveillance. Front. Cell. Infect. Microbiol. 2017, 7, 490. [CrossRef]

5. Gajadhar, A.A.; Lobanov, V.; Scandrett, W.B.; Campbell, J.; Al-Adhami, B. A novel Ehrlichia genotype detected in naturally infected cattle in North America. Vet. Parasitol. 2010, 173, 324-329. [CrossRef]

6. Lobanov, V.A.; Gajadhar, A.A.; Al-Adhami, B.; Schwantje, H.M. Molecular study of free-ranging mule deer and white-tailed deer from British Columbia, Canada, for evidence of Anaplasma spp. and Ehrlichia spp. Transbound. Emerg. Dis. 2012, 59, 233-243. [CrossRef]

7. Cabezas-Cruz, A.; Zweygarth, E.; Ribeiro, M.F.; da Silveira, J.A.; de la Fuente, J.; Grubhoffer, L.; Valdés, J.J.; Passos, L.M. New species of Ehrlichia isolated from Rhipicephalus (Boophilus) microplus shows an ortholog of the E. canis major immunogenic glycoprotein gp36 with a new sequence of tandem repeats. Parasit. Vectors 2012, 5, 291. [CrossRef]

8. Zweygarth, E.; Schöl, H.; Lis, K.; Cabezas-Cruz, A.; Thiel, C.; Silaghi, C.; Ribeiro, M.F.; Passos, L.M. In vitro culture of a novel genotype of Ehrlichia sp. from Brazil. Transbound. Emerg. Dis. 2013, 60, 86-92. [CrossRef]

9. Aguiar, D.M.; Ziliani, T.F.; Zhang, X.; Melo, A.L.; Braga, I.A.; Witter, R.; Freitas, L.C.; Rondelli, A.L.; Luis, M.A.; Sorte, E.C.; et al. A novel Ehrlichia genotype strain distinguished by the TRP36 gene naturally infects cattle in Brazil and causes clinical manifestations associated with ehrlichiosis. Ticks Tick Borne Dis. 2014, 5, 537-544. [CrossRef]

10. Carvalho, I.T.S.; Melo, A.L.T.; Freitas, L.C.; Verçoza, R.V.; Alves, A.S.; Costa, J.S.; Chitarra, C.S.; Nakazato, L.; Dutra, V.; Pacheco, R.C.; et al. Minimum infection rate of Ehrlichia minasensis in Rhipicephalus microplus and Amblyomma sculptum ticks in Brazil. Ticks Tick. Dis. 2016, 7, 849-852. [CrossRef]

11. Iweriebor, B.C.; Mmbaga, E.J.; Adegborioye, A.; Igwaran, A.; Obi, L.C.; Okoh, A.I. Genetic profiling for Anaplasma and Ehrlichia species in ticks collected in the Eastern Cape Province of South Africa. BMC Microbiol. 2017, 17, 45. [CrossRef] [PubMed]

12. Cicculli, V.; Masse, S.; Capai, L.; de Lamballerie, X.; Charrel, R.; Falchi, A. First detection of Ehrlichia minasensis in Hyalomma marginatum ticks collected from cattle in Corsica, France. Vet. Med. Sci. 2019, 5, 243-248. [CrossRef] [PubMed]

13. Rehman, A.; Conraths, F.J.; Sauter-Louis, C.; Krücken, J.; Nijhof, A.M. Epidemiology of tick-borne pathogens in the semi-arid and the arid agro-ecological zones of Punjab province, Pakistan. Transbound. Emerg. Dis. 2019, 66, 526-536. [CrossRef] [PubMed] 
14. Li, J.; Liu, X.; Mu, J.; Yu, X.; Fei, Y.; Chang, J.; Bi, Y.; Zhou, Y.; Ding, Z.; Yin, R. Emergence of a Novel Ehrlichia minasensis Strain, Harboring the Major Immunogenic Glycoprotein trp36 with Unique Tandem Repeat and C-Terminal Region Sequences, in Haemaphysalis hystricis Ticks Removed from Free-Ranging Sheep in Hainan Province, China. Microorganisms 2019, 7, 369. [CrossRef]

15. Koh, F.X.; Kho, K.L.; Kisomi, M.G.; Wong, L.P.; Bulgiba, A.; Tan, P.E.; Lim, Y.A.L.; Nizam, Q.N.H.; Panchadcharam, C.; Tay, S.T. Ehrlichia and Anaplasma Infections: Serological Evidence and Tick Surveillance in Peninsular Malaysia. J. Med. Entomol. 2018, 55, 269-276. [CrossRef]

16. Hailemariam, Z.; Krücken, J.; Baumann, M.; Ahmed, J.S.; Clausen, P.H.; Nijhof, A.M. Molecular detection of tick-borne pathogens in cattle from Southwestern Ethiopia. PLoS ONE 2017, 12, e0188248. [CrossRef]

17. Cabezas-Cruz, A.; Zweygarth, E.; Aguiar, D.M. Ehrlichia minasensis, an old demon with a new name. Ticks Tick Borne Dis. 2019, 10, 828-829. [CrossRef]

18. Meyer, D.J.; Harvey, J.W. Veterinary Laboratory Medicine: Interpretation E Diagnosis; Saunders: Philadelphia, PA, USA, 2004; ISBN 0721689264.

19. Aguiar, D.M.; Hagiwara, M.K.; Labruna, M.B. In vitro isolation and molecular characterization of an Ehrlichia canis strain from São Paulo, Brazil. Braz. J. Microbiol. 2008, 39, 489-493. [CrossRef]

20. Popov, V.L.; Han, V.C.; Chen, S.M.; Dumler, J.S.; Feng, H.M.; Andreadis, T.G.; Tesh, R.B.; Walker, D.H. Ultrastructural differentiation of the genogroups in the genus Ehrlichia. J. Med. Microbiol. 1998, 47, 235-251. [CrossRef]

21. Aguiar, D.M.; Araujo, J.P., Jr.; Nakazato, L.; Bard, E.; Cabezas-Cruz, A. Complete genome sequence of an Ehrlichia minasensis strain isolated from cattle. Microbiol. Resour. Announc. 2019, 11, 8. [CrossRef] [PubMed]

22. Seemann, T. Prokka: Rapid prokaryotic genome annotation. Bioinformatics 2014, 30, 2068-2069. [CrossRef] [PubMed]

23. Li, L.; Stoeckert, C.J., Jr.; Roos, D.S. OrthoMCL: Identification of Ortholog Groups for Eukaryotic Genomes. Genome Res. 2003, 13, 2178-2189. [CrossRef] [PubMed]

24. Ranwez, V.; Harispe, S.; Delsuc, F.; Douzery, E.J.P. MACSE: Multiple Alignment of Coding Sequences Accounting for Frameshifts and Stop Codons. PLoS ONE 2011, 6, e22594. [CrossRef] [PubMed]

25. Guindon, S.; Dufayard, J.F.; Lefort, V.; Anisimova, M.; Hordijk, W.; Gascuel, O. New Algorithms and Methods to Estimate Maximum-Likelihood Phylogenies: Assessing the Performance of PhyML 3.0. Syst. Biol. 2010, 59, 307-321. [CrossRef]

26. Gouy, M.; Guindon, S.; Gascuel, O. SeaView version 4: A multiplatform graphical user interface for sequence alignment and phylogenetic tree building. Mol. Biol. Evol. 2010, 27, 221-224. [CrossRef]

27. Zhao, Y.; Jia, X.; Yang, J.; Ling, Y.; Zhang, Z.; Wu, J.Y.J.; Xiao, J. PanGP: A tool for quickly analyzing bacterial pan-genome profile. Bioinformatics 2014, 30, 1297-1299. [CrossRef]

28. Wattam, A.R.; Davis, J.J.; Assaf, R.; Boisvert, S.; Brettin, T.; Bun, C.; Conrad, N.; Dietrich, E.M.; Disz, T.; Gabbard, J.L.; et al. Improvements to PATRIC, the all-bacterial Bioinformatics Database and Analysis Resource Center. Nucleic Acids Res. 2017, 45, D535-D542. [CrossRef]

29. Aziz, R.K.; Bartels, D.; Best, A.A.; DeJongh, M.; Disz, T.; Edwards, R.A.; Formsma, K.; Gerdes, S.; Glass, E.M.; Kubal, M.; et al. The RAST Server: Rapid annotations using subsystems technology. BMC Genom. 2008, 9, 75. [CrossRef]

30. R Core Team. R: A Language and Environment for Statistical Computing; R Foundation for Statistical Computing: Vienna, Austria, 2018; Available online: https://www.R-project.org/ (accessed on 5 September 2018).

31. Aguiar, D.M. Ehrlichiosis. In Emerging and Re-emerging Infectious Diseases of Livestock; Bayry, J., Ed.; Springer: Cham, Switzerland, 2017; pp. 365-375. [CrossRef]

32. Harrus, S.; Waner, T.; Neer, T.M. Ehrlichia canis infection. In Infectious Diseases of the Dog and Cat; Greene, C.E., Ed.; Elsevier: St. Louis, MO, USA, 2012; ISBN 9781416061304.

33. Al-Adhami, B.; Scandrett, W.B.; Lobanov, V.A.; Gajadhar, A.A. Serological cross- reactivity between Anaplasma marginale and an Ehrlichia species in naturally and experimentally infected cattle. J. Vet. Diagn. Investig. 2011, 23, 1181-1188. [CrossRef]

34. Cabezas-Cruz, A.; Vancová, M.; Zweygarth, E.; Ribeiro, M.F.; Grubhoffer, L.; Passos, L.M. Ultrastructure of Ehrlichia mineirensis, a new member of the Ehrlichia genus. Vet. Microbiol. 2013, 167, 455-458. [CrossRef] [PubMed] 
35. Cabezas-Cruz, A.; Valdés, J.J.; de la Fuente, J. The glycoprotein TRP36 of Ehrlichia sp. UFMG-EV and related cattle pathogen Ehrlichia sp. UFMT-BV evolved from a highly variable clade of E. canis under adaptive diversifying selection. Parasit Vectors 2014, 7, 584. [CrossRef] [PubMed]

36. De Fine, L.H.H. Does pathogen plasticity facilitate host shifts? PLoS Pathog. 2018, 14, e1006961. [CrossRef] [PubMed] 